\title{
The Stability of Cepheid Lightcurves
}

\author{
J.D. Fernie \\ David Dunlap Observatory, Richmond Hill, Ontario, Canada
}

\begin{abstract}
Published lightcurves of 25 to 40 day classical Cepheids and of BL Herculis stars have been examined closely for evidence of alternating deep and shallow minima, as predicted by Moskalik and Buchler (1991) and Buchler and Moskalik (1992). No such effect has been detected. Two stars, SZ Mon and EN Tra, which are sometimes taken to be classical Cepheids, do show this RV Tauri-like effect, but neither star is, in fact, likely to be a classical Cepheid.
\end{abstract}

Theoretical studies of pulsating stars by Moskalik and Buchler (1991) and by Buchler and Moskalik (1992) have predicted that BL Her stars in the approximate period range of 2.0 to 2.6 days should show alternating deep and shallow minima in their light- and velocity-curves. They predict the same effect for classical Cepheids in the 25 to 40 day range. If such an effect were present but unrealized by observers one would expect that lightcurves compiled from many different cycles would show excessive scatter in their minima. I have used existing data to search for this.

In the case of the classical Cepheids I have used the standard catalogues of Pel (1976), Moffett and Barnes (1984), and Coulson and Caldwell (1985) to search for stars with excessive scatter near minimum light. None were found. The BL Her stars had lightcurves compiled from many different sources covering many years, but again no unusual effects near minimum light were found.

Two stars, SZ Mon and EN Tra, were found to show RV Tauri-like behaviour. However, the galactic latitude of EN Tra would place it $900 \mathrm{pc}$ below the galactic plane if it were a classsical Cepheid, while previous discussions (Stobie 1970; Lloyd Evans 1971) have already established that SZ Mon is not a classical Cepheid. Both stars show large far ir excesses (McAlary and Welch 1986), something not seen in classical Cepheids.

\section{References:}

Buchler, J.R., and Moskalik, P. 1992, ApJ, 391, 736.

Coulson, I.M., and Caldwell, J.A.R. 1985, So. Afr. Ast. Obs. Circ. No. 9, 1.

Lloyd Evans, T. 1971, The Observatory, 91, 159.

McAlary, C.W., and Welch, D.L. 1986, AJ, 91, 1209.

Moffett, T.J., and Barnes III, T.G. 1984, ApJS, 55, 389.

Moskalik, P., and Buchler, J.R. 1991, ApJ, 366, 300.

Pel, J.W. 1976, A\&AS, 24, 413.

Stobie, R.S. 1970, MNRAS, 148, 1. 